\title{
The quality of blood dropped from an unmanned aerial vehicle (Drone)
}

\begin{abstract}
Objective: To evaluate the risk of deterioration in the quality of blood transported by a fixed-wing drone.

Materials and methods: Red blood cell (RBC) solutions were categorized into "travel group" (transported by train or car to test place) and "non-travel" group. The travel group was further subcategorized into "dropped," and "non-dropped" groups. One bag each from the dropped and non-dropped groups were wrapped in an Absorption Sheet (A-Sheet) and an Under Sheet (U-Sheet). RBC solution of dropped group was dropped onto the ground from an altitude of $10 \mathrm{~m}$. Lactate dehydrogenase (LD) levels from the samples of RBC solution were measured and the LD ratio (LDR) was calculated as the rate of increase of the LD which can be compared. We compared the differences in LDR between the dropped and non-dropped groups.
\end{abstract}

Results: In the dropped group, there was an increase in LDR before filtration in the blood wrapped in the A-sheet. No increase was noted in the blood wrapped in the U-sheet. LDR levels after filtration were slightly increased in the blood samples wrapped in the U-sheet and clearly increased in blood samples wrapped in the A-Sheet.

Conclusion: We considered the possibility of blood getting hemolyzed due to a fall and observed that hemolysis was affected by packing. Thus, since it may be difficult to find good packaging for blood, dropped blood may not be safe for transfusion due to the likelihood of hemolysis. Hence, for practical purposes, using a drone without a fixed-wing may be preferable for transportation of blood

Keywords: blood, unmanned aerial vehicle, drone, red blood cells, active transport refrigerator, lactate dehydrogenase ratio
Volume 8 Issue 2 - 2020

\author{
Koki Yakushiji,' Fumiatsu Yakushiji, ${ }^{2,3}$ Hiroshi \\ Fujita $^{4}$ \\ 'Faculty of Policy Management, Keio University, Japan \\ ${ }^{2}$ Department of Internal Medicine, Tokyo Metropolitan Bokutoh \\ Hospital, Japan \\ ${ }^{3}$ Faculty of Medicine, Toho University, Japan \\ ${ }^{4}$ Department of Transfusion Medicine, Tokyo Metropolitan \\ Bokutoh Hospital, Japan
}

Correspondence: Fumiatsu Yakushiji, MD, PhD, Department of Internal Medicine, Tokyo Metropolitan Bokutoh Hospital, 4-23-15 Koutoubashi Sumida, Tokyo 130-8575, Japan, Tel +8I336336I5I, Fax 8I-3-3633-6I73, Email clinic@nifty.com

Received: April II, 2020 | Published: April 30, 2020
Abbreviations: RBC, red blood cell; A-sheet, absorption sheet; U-sheet, under sheet; LD, lactate dehydrogenase; LDR, LD ratio

\section{Dear editor}

Transportation of blood products using an unmanned aerial vehicle is being conducted in Rwanda and other places. ${ }^{1,2}$ Blood is dropped onto the ground by an airborne drone, and transportation by a fixedwing drone may result in considerable shock to the blood. Although blood is known to hemolyze through external force and vibration, we confirmed that there was no hemolysis of blood transported by ship at the start of the Ogasawara blood rotation. ${ }^{4}$ We believe that a parachute should be attached to reduce the shock when dropping the blood. However, we consider that dropping blood using a parachute may not reduce the shock sufficiently. We have previously reported safe transportation of blood by quadcopter, which differs from a drone with a fixed wing. ${ }^{5,6}$ However, the method of dropping blood from a fixed-wing drone has not been evaluated yet.

We believe that the employed method of blood transportation may result in hemolysis of the transported blood.

In the laboratory, we have experienced that the drops from a height of $1 \mathrm{~m}$ can damage the blood bags, but not always. Further, as a preliminary experiment, no abnormality was observed in the $3 \mathrm{~m}$ drop experiment. As the height of the three-story building is about 10 $\mathrm{m}$, we considered that the flying height of the drone is more than 10 $\mathrm{m}$. Therefore, we examined the damage to the blood dropped to the ground from a height of $10 \mathrm{~m}$.

We reapportioned two bags of irradiated red blood cells (RBC) solution (RBC \#1 and \#2) collected from an in-house blood disposal unit into three bags (approximately $93 \mathrm{~mL}$ each). We categorized the two bags into the travel and non-travel groups. The travel group was subcategorized into the dropped group (\#11, \#21), transported by a drone; and the non-dropped group (\#12,\#22). The blood was kept in active transport refrigerator (ATR) (FUJIFILM Toyama Chemical Co. Ltd., Tokyo) and transported by train or car to Robot Test Field located in Fukushima prefecture. The blood in non-travel groups (control, $\# 13, \# 23$ ) was stored at $4.0 \pm 0.3^{\circ} \mathrm{C}$ in our laboratory. We wrapped \#11 and \#12 in an Absorption Sheet (A-Sheet, Hakujuji Co. Ltd., Tokyo; size $45 \times 60 \mathrm{~cm}$ ), and the others (\#21, \#22) in an Under Sheet (U-Sheet, Koyo Co. Ltd., Tokyo; $60 \times 90 \mathrm{~cm})$. We lifted \#11 and \#21 using a quadcopter, M1000 (Mazex Co. Ltd., Osaka), to an altitude of $10 \mathrm{~m}$, following which they were dropped from the air directly onto the ground. We used quadcopter instead of an airborne drone because the latter could be not be operated at the test place.

In our study evaluating the effect of transportation by car on blood for transfusion, lactate dehydrogenase (LD) was the most sensitive indicator for degradation of $\mathrm{RBCs}^{7}{ }^{7}$ therefore, $\mathrm{LD}$ was used for evaluation in the present study. Samples were taken from the RBC solutions on the day of experiment and two days later. LD levels were 
measured before and after the travel (before and after filtration), and the LD ratio (LDR) was calculated as follows:

$$
\frac{\text { LD level (travel group) after travel/LD level (travel group) before the experiment }}{\text { LD level (no - travel group) after travel group's travel/LD level (no - travel group) before the experiment }}
$$

We compared the differences in LDR between the dropped and non-dropped samples. The differences were analyzed using the Student's t-test. Significance level was set at $p<0.05$. Data are expressed as group mean \pm standard deviation $(\mathrm{N}=4)$.

In this letter, we report the results of the experiment performed at the Fukushima Robot Test Field, where there were no restrictions on

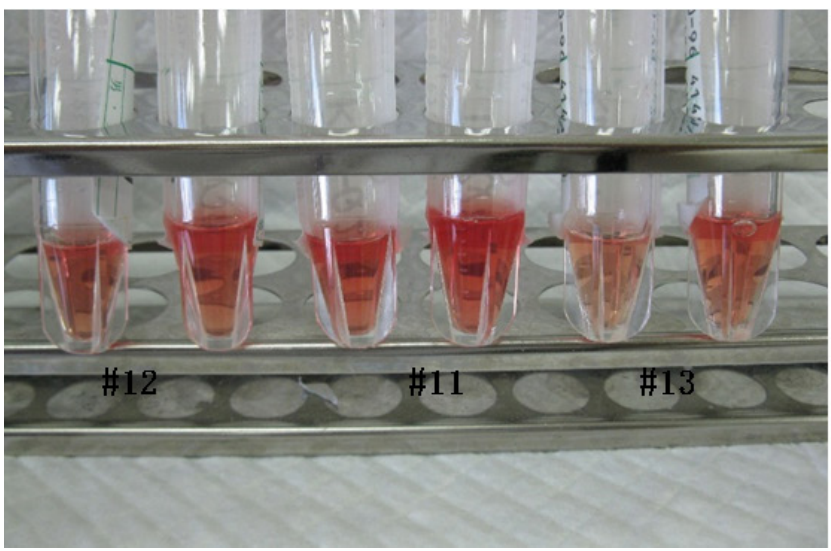

the experiment, and the Japanese laws were obeyed while conducting the experiment.

No apparent damage to the bags was observed after being dropped. However, macroscopic hemolytic changes were observed in the blood sample in the bag wrapped in the A-Sheet (Figure 1).

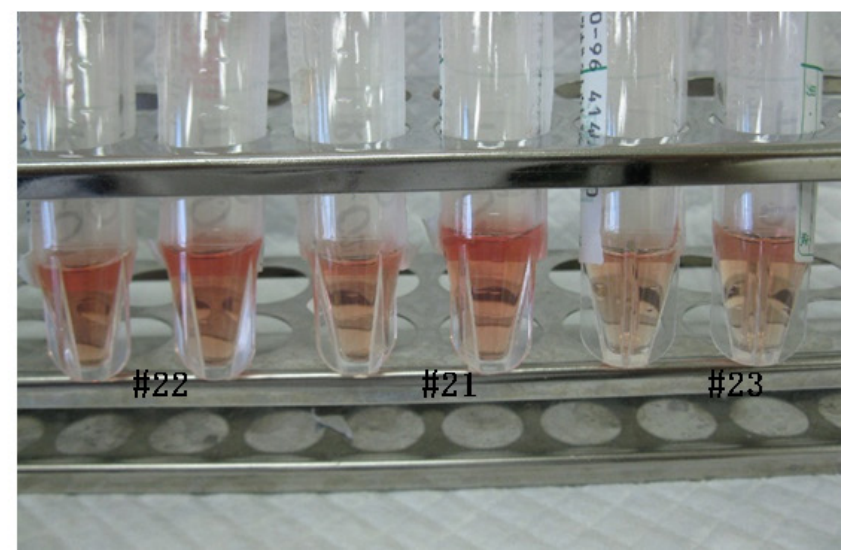

After filtration, stronger hemolysis occurred in the dropped RBC sample (\#II) wrapped in A-sheet than that in the non-dropped group (\#I2).

$\# 21$ and \#22 were wrapped in U-sheet.

$\# 13$ and \#23 were control samples (non-travel group).

A-sheet, Absorption Sheet; U-sheet, Under Sheet.

Figure I Macroscopic RBC solution, after travel and post-filtration.

The LDR before filtration of the dropped group wrapped in the U-Sheet $(1.37 \pm 0.02$ fold) was lower than that of the non-dropped group ( $1.49 \pm 0.03$ fold), while the ratio of dropped group wrapped in the A-Sheet (1.63 \pm 0.03 -fold) was higher than that in the nondropped group (1.42 \pm 0.03 -fold). After filtration, it was clear that

there was damage due to the drop $(1.74 \pm 0.08$-fold vs. $1.44 \pm 0.02$ fold for A-Sheet; $1.45 \pm 0.02$-fold vs. $1.40 \pm 0.02$-fold for the U-Sheet). Transportation of blood by car or train may cause decrease in blood quality as well. Moreover, our data also showed damage to the blood as a result of dropping it from a drone (Table 1).

Table I Comparison of lactate dehydrogenase (LD) levels (IU/L) in red blood cell solution between the I0-m drop group and control group (no travel, no dropping)

\begin{tabular}{llll}
\hline & Before experiment & After travel & Post-filtration** \\
\hline RBC \# I* & & & \\
LDH (IU/L) & & & \\
Non-travel group (without travel) & 185.01 .8 & $232.8 \pm 6.6$ & $277.8 \pm 7.4$ \\
Non-dropped group (with travel) & $216.0 \pm 5.3$ & $307.0 \pm 3.7$ & $310.8 \pm 3.6$ \\
Dropped group (I0 m drop using drone) & $214.5 \pm 4.8$ & $350.5 \pm 6.2$ & $375.3 \pm 5.0$ \\
LDR (fold) & & & \\
Non-travel group (without travel) & & $1.26 \pm 0.04$ & $1.50 \pm 0.04$ \\
Non-dropped group (with travel) & & $1.42 \pm 0.03$ & $1.44 \pm 0.02$ \\
Dropped group (I0 m drop using a drone) & $1.63 \pm 0.03$ & $1.74 \pm 0.08$ \\
P-value of LDR; dropped group vs. non-travel group & $<0.000$ & $<0.000$ \\
P-value of LDR; dropped group vs. non-dropped group & $<0.000$ & $<0.000$ \\
\hline
\end{tabular}


Table Continued...

\begin{tabular}{llll}
\hline & Before experiment & After travel & Post-filtration** \\
\hline RBC\#2* & & & \\
LDH (IU/L) & & & \\
Non-travel group (without travel) & $226.8 \pm 11.6$ & $307.8 \pm 20.3$ & $275.5 \pm 4.8$ \\
Non-dropped group (with travel) & $251.3 \pm 7.4$ & $343.8 \pm 5.9$ & $365.5 \pm 4.0$ \\
Dropped group (I0 m drop using drone) & $263.8 \pm 22.7$ & $392.5 \pm 8.8$ & $370.0 \pm 4.8$ \\
LDR (fold) & & & \\
Non-travel group (without travel) & & $1.35 \pm 0.04$ & $1.21 \pm 0.02$ \\
Non-dropped group (with travel) & & $1.49 \pm 0.03$ & $1.40 \pm 0.02$ \\
Dropped group (I0 m drop using a drone) & $1.37 \pm 0.02$ & $1.45 \pm 0.02$ \\
P-value of LDR; dropped group vs. non-travel group & & 0.4 & $<0.000$ \\
P-value of LDR; dropped group vs. non-dropped group & $<0.001$ & $<0.001$ \\
\hline
\end{tabular}

LD, lactate dehydrogenase; LDR, lactate dehydrogenase ratio

*RBC \#I was wrapped in Absorption Sheet; RBC \#2 was wrapped in Under Sheet

**Post-filtration implies that the RBC solution was passed through a transfusion filter (Terumo Co., Tokyo) used in transfusion practice

LDR= LD level post-travel or post-filtration/Average LD level of RBC solution before the experiment. Unit: fold

P-value was calculated using the Student's $t$-test. Significance level was set at $<0.05$

Data are expressed as group mean \pm standard deviation $(\mathrm{N}=4)$

We considered the possibility of blood getting hemolyzed due to a fall and observed that hemolysis was affected by packing material as well. Thus, since it may be difficult to find good packaging for blood, dropped blood may not be safe for transfusion due to the likelihood of hemolysis. Hence, for practical purposes, using a drone without a fixed-wing may be preferable.

In addition, we observed hemolysis in the blood that had been accidentally dropped from a multicopter in the past. That accident and the trial reported here suggest that dropped blood should not be used for transfusion. Therefore, we cannot recommend that the method of dropping blood from fixed-wing drones, as practiced in Africa, should be adopted in Japan as well.

\section{Acknowledgments}

None.

\section{Conflicts of interest}

The authors declare no conflicting interests.

\section{References}

1. Amukele T, Ness PM, Tobian AAR, et al. Drone transportation of blood products. Transfusion. 2017;57(3):582-588.

2. Glauser W. Blood-delivering drone saving lives in Africa and maybe soon in Canada. CMAJ. 2018;190(3):E88-E89.

3. Faghih MM, Sharp MK. Modeling and prediction of flow-induced hemolysis: a review. Biomech Model Mechanobiol. 2019;18(4):845-881.

4. Igarashi T, Fujita H. Asaka H, et al. Patient rescue, and blood utilization in Ogasawara blood rotation system. Transfusion. 2018;58(3):788-794.

5. Yakushiji K, Yakushiji F, Fujita H. Initial trial on the transport of blood products using an unmanned aerial vehicle (UAV) in Japan in preparation for disaster. Hematol Transfus Int J. 2019;7(3):77-78.

6. Yakushiji F, Yakushiji K. Murata M, et al. The quality of blood is not affected by drone transport: an evidential study of the unmanned aerial vehicle conveyance of transfusion material in Japan. Drones. 2020;4(1):4.

7. Fujita H, Tojo Y, et al. Temperature management of red blood cell solution transported by car for transfusion at home. Open J Blood Dis. 2020;10:37-40. 